\title{
A CMOS BANDGAP REFERENCE CIRCUIT FOR SUB-1-V OPERATION WITHOUT USING EXTRA LOW-THRESHOLD-VOLTAGE DEVICE
}

\author{
Ming-Dou Ker, Jung-Sheng Chen, and Ching-Yun Chu \\ Nanoelectronics \& Gigascale Systems Laboratory \\ Institute of Electronics, National Chiao-Tung University, Taiwan
}

\begin{abstract}
A new sub-1-V CMOS bandgap voltage reference without using low threshold voltage device is presented in this paper. The new proposed sub-1-V bandgap reference with startup circuit has been successfully verified in a standard $0.25 \mu \mathrm{m}$ CMOS process. The experimental results show that, at the minimum supply voltage of $0.85 \mathrm{~V}$, the output reference voltage is $238.2 \mathrm{mV}$ with an effective temperature coefficient of $58.1 \mathrm{ppm} /{ }^{\circ} \mathrm{C}$ while DC current is $28 \mu \mathrm{A}$. At $0.85 \mathrm{~V}$ supply voltage, the measured power noise rejection ratio is $-33.2 \mathrm{~dB}$ at $10 \mathrm{kHz}$.
\end{abstract}

\section{INTRODUCTION}

Low voltage and low power are two important design criteria in both analog and digital systems. It is expected that the whole system will be operated down to a single $1 \mathrm{~V}$ supply in near future. The bandgap reference generators which can operate from $1 \mathrm{~V}$ supply are widely used in DRAM and flash memories.

In CMOS technology, parasitic vertical bipolar junction transistors (BJT) are used in high-precision bandgap voltage reference. The conventional CMOS bandgap reference does not work in near $1 \mathrm{~V}$ supply voltage. The reason, that the minimum supply voltage can not be lower than $1 \mathrm{~V}$, is constrained by two factors. One is that the output voltage is around $1.25 \mathrm{~V}$ [1], [2], which exceeds $1 \mathrm{~V}$ supply. The other is that the low-voltage design of the proportional to absolute temperature current generation loop is limited by the input common-mode voltage of the amplifier [1], [3]. These two limitations can be solved by the resistive subdivision methods [4], [5], using low threshold voltage (or native) device [4]-[6], BiCMOS process [3], or DTMOST [7] device. However, either low threshold devices or BiCMOS process will increase the fabrication cost to IC products.

In this work, a new bandgap reference is proposed, which can be successfully operated with sub-1-V supply in a standard $0.25 \mu \mathrm{m}$ CMOS process without special process technology. The new proposed bandgap voltage reference with a stable output voltage $\mathrm{V}_{\mathrm{REF}}$ of $238.2 \mathrm{mV}$ and temperature coefficient of $58.1 \mathrm{ppm} /{ }^{\circ} \mathrm{C}$ under VDD power supply of $0.85 \mathrm{~V}$ has been proven in the silicon chip.

\section{CONVENTIONAL BANDGAP REFERENCE CIRCUIT}

A typical CMOS implementation of a bandgap reference is shown in Fig. 1. The output reference voltage $V_{\text {REF }}$ of the conventional bandgap reference circuit can be written as

$$
V_{R E F-C O N V}=V_{E B 2}+\frac{R_{2}}{R_{1}} V_{T} \ln \left(\frac{A_{1}}{A_{2}}\right)
$$

where $A_{1}$ and $A_{2}$ is the emitter areas of $Q_{1}$ and $Q_{2}$, and $V_{T}$ is thermal voltage. The second term in (1) is proportional to the absolute temperature (PTAT), which is used to cancel the negative temperature coefficient of $\mathrm{V}_{\mathrm{EB}}$. Hence, if a proper ration of resistors is kept, an output voltage with low sensitivity to the temperature can be obtained. In general, the $\mathrm{V}_{\mathrm{REF}}$ is about $1.25 \mathrm{~V}$, so that the conventional bandgap reference circuit can not be used in low voltage application, such as $1 \mathrm{~V}$.

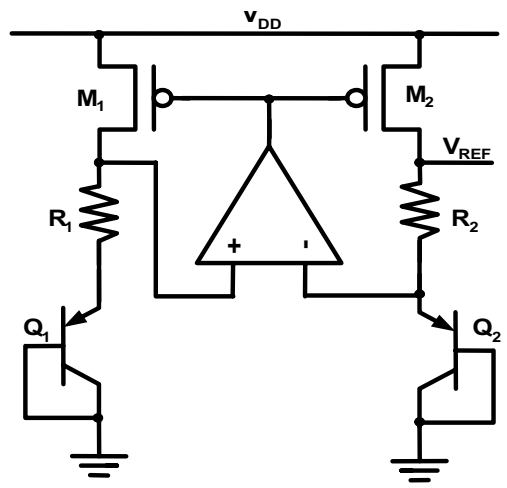

Fig. 1. The conventional bandgap reference circuit.

\section{NEW PROPOSED BANDGAP REFERENCE CIRCUIT}

The concept of the new proposed bandgap reference is that the two voltages (which are proportional to $\mathrm{V}_{\mathrm{EB}}$ and $\mathrm{V}_{\mathrm{T}}$ ) are generated by only one feedback loop. The two-stage operational amplifier with p-channel input is used in this new proposed bandgap reference.

The new proposed bandgap reference is shown in Fig. 2, which uses the resistive subdivision $R_{1 a}, R_{1 b}, R_{2 a}$ and $R_{2 b}$ to 
reduce the input common-mode voltage of the amplifier. The dimensions of PMOS devices $\mathrm{M}_{1}$ and $\mathrm{M}_{2}$ are the same. The resistance of $R_{1 a}$ and $R_{2 a}$ is the same, and the resistance of $R_{1 b}$ and $R_{2 b}$ is the same. Following the KCL at the nodes of $V_{1}$ and $V_{2}$ in Fig. 2, the reference voltage can be expressed as

$$
\begin{aligned}
V_{R E F} & =\frac{R_{1 b}}{R_{1 a}+R_{1 b}}\left[\left(R_{4}+\frac{R_{1 a} R_{1 b}}{R_{1 a}+R_{1 b}}\right) \frac{\Delta V_{E B}}{R_{3}}+V_{E B 2}\right] \\
& =\frac{R_{1 b}}{R_{1 a}+R_{1 b}}\left[V_{R E F-\text { CONV }}^{*}+\left(\frac{R_{1 a} R_{1 b}}{R_{1 a}+R_{1 b}}\right) \frac{\Delta V_{E B}}{R_{3}}\right]
\end{aligned}
$$

where $\Delta V_{E B}=V_{E B 2}-V_{E B 1}$. The term of $V_{R E F-C O N V}^{*}$ in (2) should be noted that its representation is identical to the conventional reference voltage in (1), but the actual value is not exactly the same as the conventional one, since the term of $\left(\frac{R_{1 a} R_{1 b}}{R_{1 a}+R_{1 b}}\right) \frac{\Delta V_{E B}}{R_{3}}$ is still a temperature dependent factor. The minimum supply voltage of the new proposed bandgap reference is reduced effectively as

$$
V_{D D(\text { Min })}=V_{R E F}+\left|V_{T P}\right|+2\left|V_{D S s a t}\right| .
$$

The new proposed bandgap reference can be operated near sub-1-V supply voltage.

\section{CIRCUIT IMPLEMENTATION}

The whole complete circuit to realize the proposed sub-1-V bandgap reference is shown in Fig. 3. The circuit is composed of a bias circuit, a bandgap core, two startup circuits, and a two-stage operational amplifier. The bandgap reference circuit has two stable points. To ensure that it ends up to the correct state, a startup circuit must be added. There should be a startup circuit for the bias circuit to avoid the bias circuit working in zero-current state. The startup circuit for the bias circuit of the proposed bandgap reference is formed by $\mathrm{M}_{\mathrm{S1a}} \sim \mathrm{M}_{\mathrm{S} 3 \mathrm{a}}$. Similarly, another startup circuit is used to ensure that the input voltage of the amplifier is not kept at zero in the initial state. The capacitors $\mathrm{C}_{1}$ and $\mathrm{C}_{2}$ are used to stabilize the circuit. The bulk and the source of the input pair transistors $\mathrm{M}_{\mathrm{A} 2}$ and $\mathrm{M}_{\mathrm{A} 3}$ in the amplifier should be connected together to make the threshold voltage lower without the body effect.

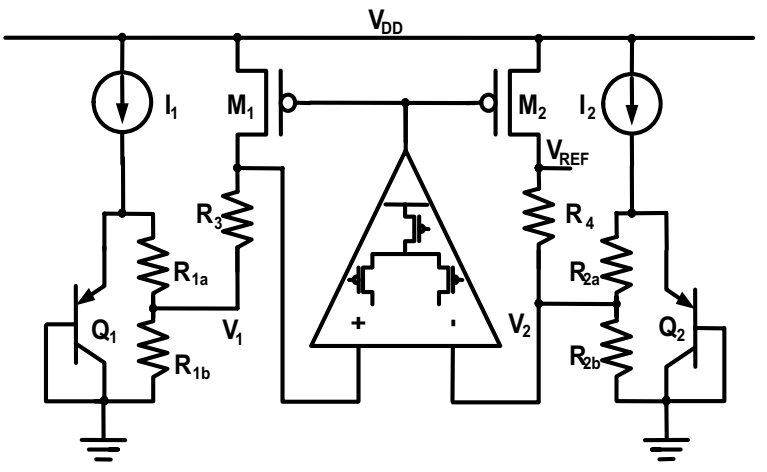

Fig. 2. The new proposed bandgap reference circuit.

Another important factor of the bandgap reference is the power noise rejection ratio (PSRR) [8], which represents the resistance against the noise from the supply voltage in the unit of decibel $(\mathrm{dB})$. The small signal model of the proposed bandgap reference with a p-channel input operational amplifier is shown in Fig. 4. The resistance $R_{3}^{\prime}$ and $R_{4}^{\prime} \quad$ in Fig. 4 are $R_{3}+\left(R_{1 a} / / R_{1 b}\right)$ and $R_{4}+\left(R_{2 a} / / R_{1 b}\right)$, respectively. The capacitor $\mathrm{C}$ is the parasitic drain-to-bulk junction capacitance of $\mathrm{M}_{2}$. Since the turn-on resistances of the PNP transistors are small, both $\mathrm{Q}_{1}$ and $\mathrm{Q}_{2}$ can be considered as short-circuit to the ground.

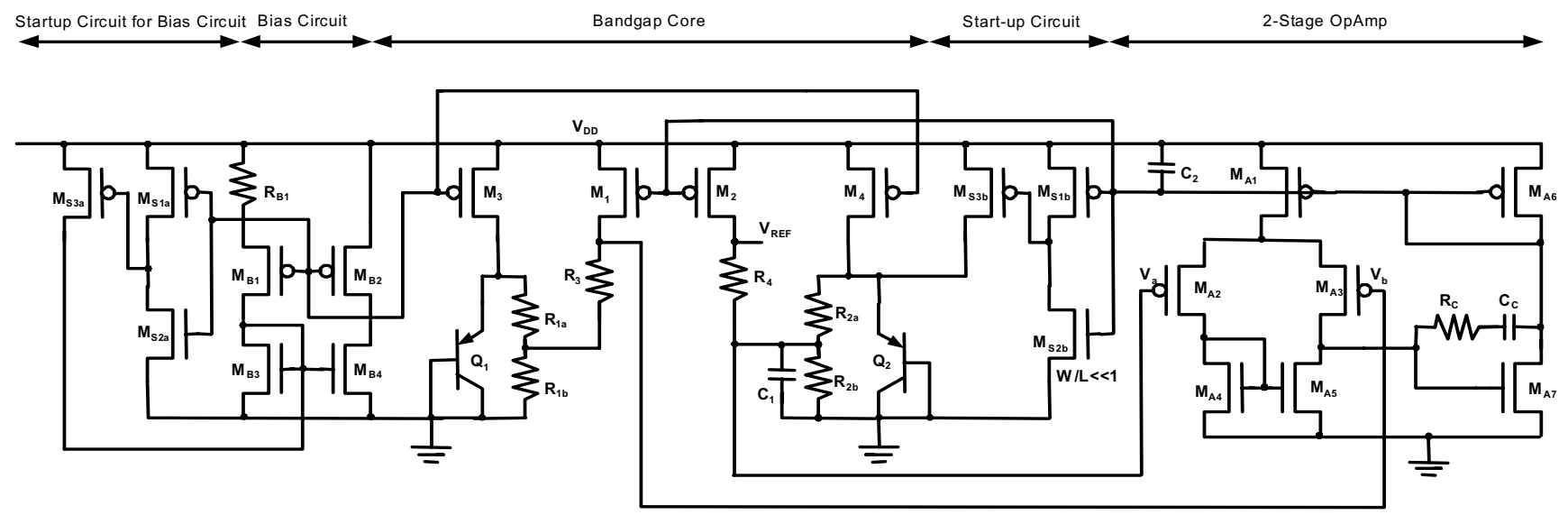

Fig. 3. Complete schematic of the new proposed bandgap reference. 
The amplifier is modeled with an output resistor $R_{\text {out }}$, and two voltage-controlled current sources $i_{d}$ and $i_{d d}$, driven by the differential input voltage $\mathrm{v}_{\mathrm{d}}$, and power supply voltage $v_{d d}$, respectively. The ratio of the output reference voltage and the noise from the supply voltage can be expressed as

$$
\frac{V_{R E F}(s)}{V_{D D(\text { Noise })}(s)}=\frac{1}{s C r_{o 2}+\frac{r_{o 2}}{R_{4}^{\prime}}+1} .
$$

The PSRR of the proposed bandgap reference will become worse at high frequency. It is apparent that changing the capacitance at $\mathrm{V}_{\mathrm{REF}}$ node can shift the pole location. Moving the pole to the left in the s-plane will result in an improvement in high frequency noise rejection. This can be achieved by inserting a capacitance $C_{p}$ to ground at $\mathrm{V}_{\mathrm{REF}}$ node. Thus, (4) is changed to (5) as below

$$
\frac{V_{R E F}(s)}{V_{D D(\text { Noise })}(s)}=\frac{1}{s\left(C+C_{p}\right) r_{o 2}+\frac{r_{o 2}}{R_{4}^{\prime}}+1} \text {. }
$$

A larger $C_{p}$ provides better stability, but the startup time will be longer.

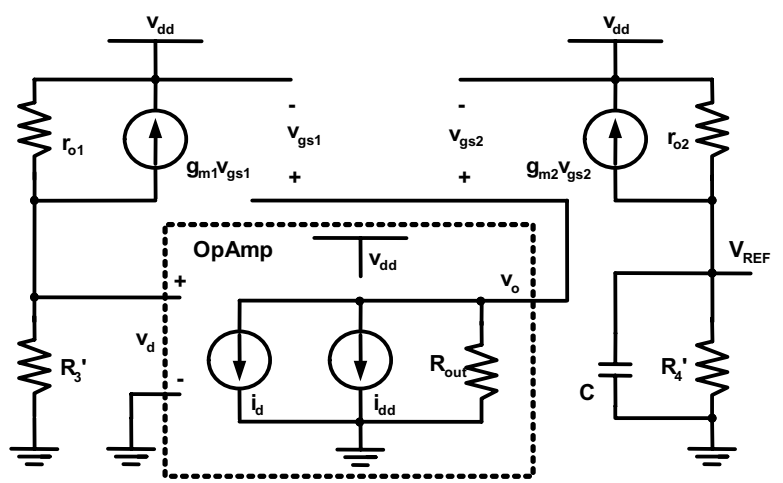

Fig. 4. Small signal model of the proposed bandgap reference.

\section{EXPERIMENTAL RESULTS}

\subsection{Simulation}

The proposed bandgap reference has been simulated by varying its operating temperature from 0 to $100{ }^{\circ} \mathrm{C}$. The power supply voltage was set from 0.8 to $1.2 \mathrm{~V}$. The simulated results are shown in Fig. 5. The temperature coefficient is around $75 \mathrm{ppm} /{ }^{\circ} \mathrm{C}$ with the supply voltage at $0.85 \mathrm{~V}$. With the supply voltage at $0.8 \mathrm{~V}$, the temperature coefficient grows sharply to be above $200 \mathrm{ppm} /{ }^{\circ} \mathrm{C}$. Fig. 6 shows the output reference voltage with the supply voltage varied from 0 to $1.5 \mathrm{~V}$. The curves of output reference voltages under $0{ }^{\circ} \mathrm{C}, 25^{\circ} \mathrm{C}$ and $100{ }^{\circ} \mathrm{C}$ grow together while the supply voltage of the proposed bandgap reference is at
0.85 V. Fig. 7 shows the PSRR with different $C_{p}$ at 0 pF, 0.1 $\mathrm{pF}, 0.2 \mathrm{pF}$, and $0.5 \mathrm{pF}$. The PSRR at low frequency of the proposed bandgap reference, which works in low supply voltage at $0.85 \mathrm{~V}$, is $-30.2 \mathrm{~dB}$. When the temperature grows to $100{ }^{\circ} \mathrm{C}$, the PSRR will decrease at high temperature. Moving the pole to the left in the s-plane can result in an improvement in high frequency noise rejection.

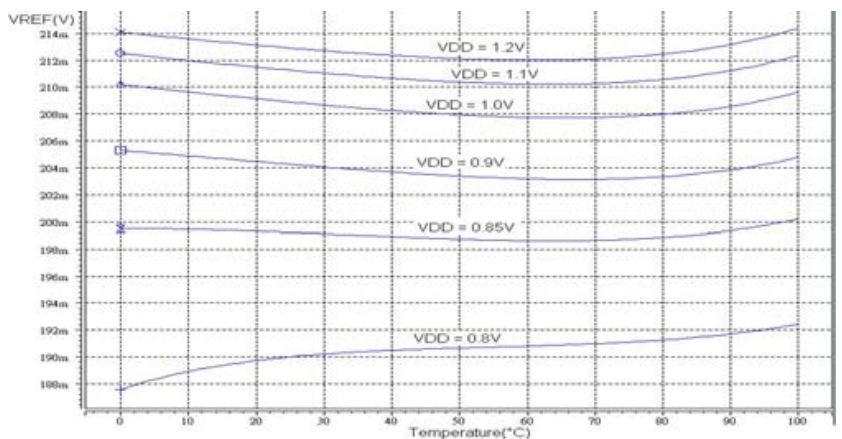

Fig. 5. Simulated reference voltage of the proposed bandgap reference with different supply voltages.

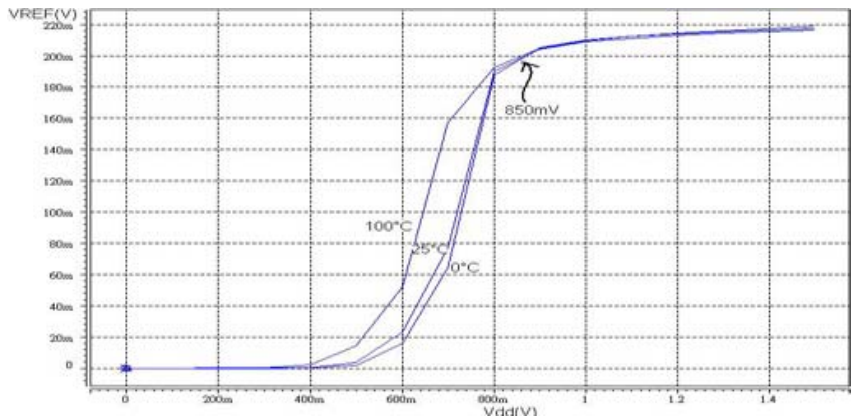

Fig. 6. Simulated minimum supply voltage of the proposed bandgap reference.

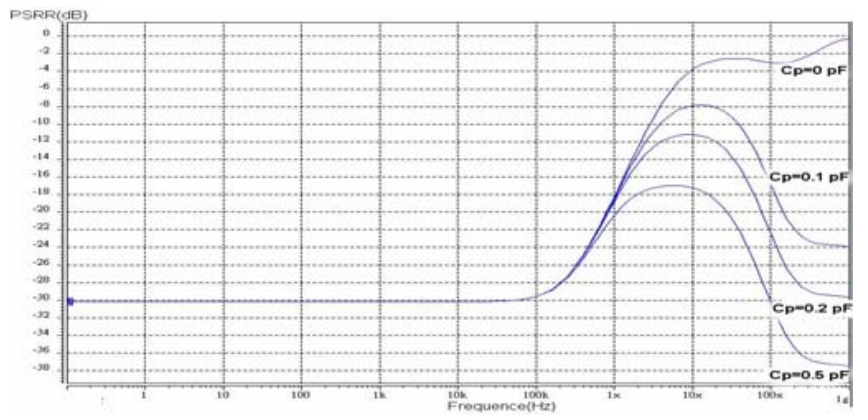

Fig. 7. Simulated PSRR of the proposed bandgap reference with different capacitances of $\mathrm{C}_{\mathrm{p}}$.

\subsection{Silicon Verification}

The proposed bandgap reference has been fabricated in a $0.25 \mu \mathrm{m}$ single-ploy-five-metal (1P5M) CMOS technology. The proposed bandgap reference consists of the bandgap core, bipolar transistors, and resistors. Fig. 8 shows the overall die photo of the new sub-1-V bandgap reference. The occupied silicon area of the new proposed bandgap 
reference is only $177 \mu \mathrm{m} \times 106 \mu \mathrm{m}$. The bandgap reference has been measured by varying its operating temperature from -10 to $120^{\circ} \mathrm{C}$. The power supply voltage was set from 0.8 to $1.2 \mathrm{~V}$. The measured results are shown in Fig. 9. The temperature coefficient is around $58.1 \mathrm{ppm} /{ }^{\circ} \mathrm{C}$ with the supply voltage at $0.85 \mathrm{~V}$. The experimental results in Fig. 10 have confirmed that the minimum supply voltage for the sub-1-V bandgap reference is $0.85 \mathrm{~V}$. The measured power noise rejection ratio is $-33.2 \mathrm{~dB}$ at $10 \mathrm{kHz}$, and output reference voltage is $238.2 \mathrm{mV}$ under the VDD power supply of $0.85 \mathrm{~V}$.

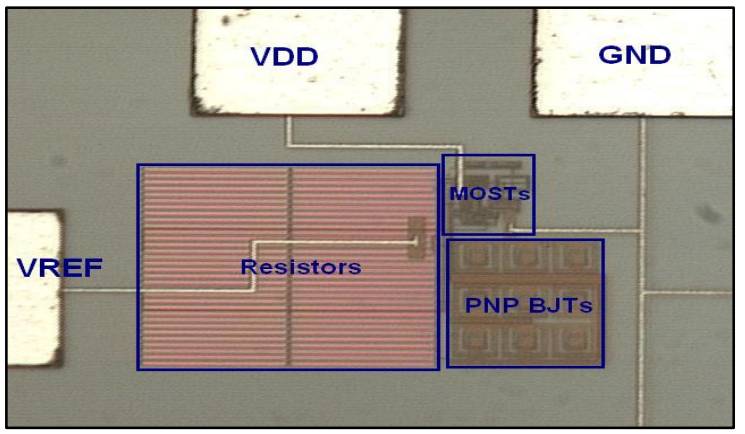

Fig. 8. Die photo of the new proposed bandgap reference.

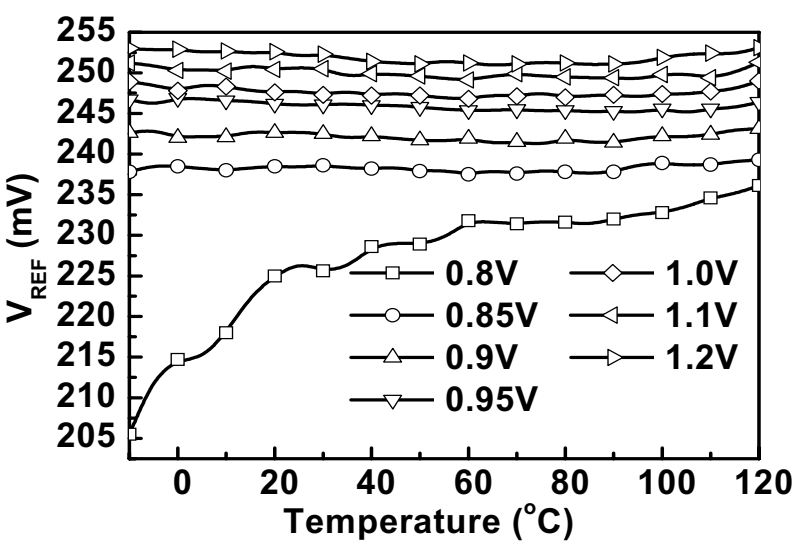

Fig. 9. Dependence of output reference voltage on the temperature under different VDD voltage levels.

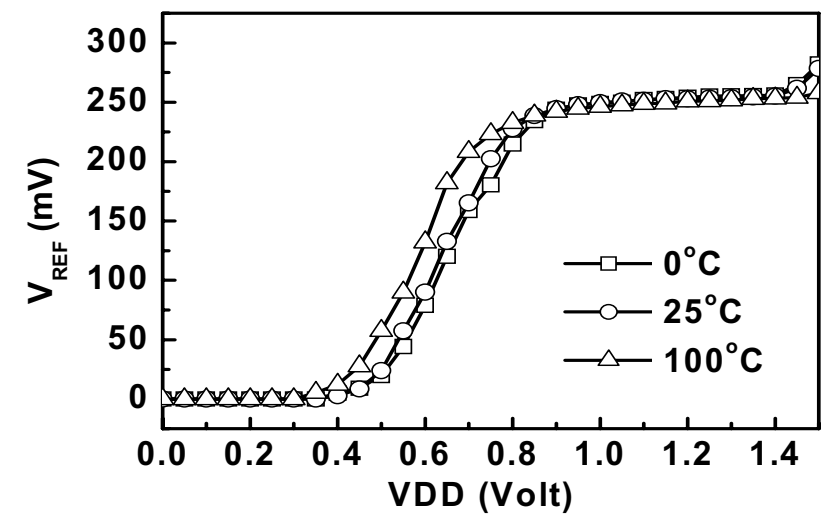

Fig. 10. Dependence of output reference voltage on the VDD supply voltage under different temperatures.

\section{CONCLUSION}

A CMOS bandgap voltage reference with $\mathrm{V}_{\mathrm{REF}}$ of 238.2 $\mathrm{mV}$ and temperature coefficient of $58.1 \mathrm{ppm} /{ }^{\circ} \mathrm{C}$, which consumes a maximum current of $28 \mu \mathrm{A}$ at $0.85 \mathrm{~V}$ supply, has been presented. The sub-1-V operation of the bandgap reference has been successfully achieved in this work without using the low-threshold-voltage devices. Moreover, other techniques to achieve sub-1-V operation, such as low voltage startup circuit and the lower common-mode input range of the amplifier by resistive subdivision, have been described. Without using low-threshold-voltage device, the proposed bandgap reference circuit can be implemented in general CMOS technology.

\section{REFERENCES}

[1] Y. Jiang and E. K. F. Lee, "Design of low-voltage bandgap reference using transimpedance amplifier," IEEE Trans. Circuits Syst. II, vol. 47, pp. 552-555, June 2000.

[2] K. N. Leung and K. T. Mok, “A sub-1-V 15-ppm $/{ }^{\circ} \mathrm{C}$ CMOS bandgap reference without requiring low threshold voltage device," IEEE J. Solid-State Circuit, vol. 37, pp. 526-529, April 2002.

[3] P. Malcovati, F. Maloberti, M. Pruzzi, and C. Fiocchi, "Curvature compensated BiCMOS bandgap with $1-\mathrm{V}$ supply voltage," IEEE J. Solid-State Circuit, vol. 36, pp.1076-1081, July 2001.

[4] H. Neuteboom, B. M. J. Kup, and M. Janssens, "A DSP-based hearing instrument IC," IEEE J. Solid-State Circuits, vol. 32, pp. 1790-1806, Nov. 1997.

[5] H. Banba, H. Shiga, A. Umezawa, T. Miyaba, T. Tanzawa, S. Atsumi, and K. Sakui, "A CMOS bandgap reference circuit with sub-1-V operation," IEEE J. Solid-State Circuits, vol. 34, pp. 670-674, May 1999.

[6] G. Giustolisi, "A low-voltage low-power voltage reference based on subthreshold MOSFETs," IEEE $J$. Solid-State Circuits, vol. 38, pp. 151-154, Jan. 2003.

[7] A.-J. Annema, "Low-power bandgap references featuring DTMOSTs," IEEE J. Solid-State Circuits, vol. 34, pp. 949-955, July 1999.

[8] G. Giustolisi and G. Palumbo, "A detailed analysis of power-supply noise attenuation in bandgap voltage references," IEEE Trans. on Circuits and Systems-I: Fundamental Theory and Applications, vol. 50, no. 2, pp. 185-197, Feb. 2003. 